\title{
Myomectomy in pregnancy: feasibility and safety
}

\section{Reddi Rani P., Ashwini Vishalakshi L.*, Lopamudra B. John}

Department of Obstetrics and Gynecology, Mahatma Gandhi Medical College and Research Institute, Pilliyarkuppam, Pondicherry, India

Received: 08 August 2017

Revised: 01 September 2017

Accepted: 04 September 2017

\section{*Correspondence:}

Dr. Ashwini Vishalakshi L., E-mail: akshivishal@gmail.com

Copyright: () the author(s), publisher and licensee Medip Academy. This is an open-access article distributed under the terms of the Creative Commons Attribution Non-Commercial License, which permits unrestricted non-commercial use, distribution, and reproduction in any medium, provided the original work is properly cited.

\begin{abstract}
Prevalence of myoma in pregnancy is increasing due to advances in imaging technology. Majority are asymptomatic. Symptomatic myomas are usually large, increase in size during pregnancy and give rise to various obstetrical complications. Myomectomy during pregnancy is controversial. The management of fibroids encountered during pregnancy and caesarean section is a therapeutic dilemma. Myomectomy during pregnancy and caesarean section is discouraged traditionally due to fear of miscarriage, uncontrolled bleeding, failure to obliterate the cavity, and ending in hysterectomy. Recent literature suggests myomectomy during pregnancy and caesarean section is safe in well selected cases with experienced obstetrician in a tertiary care center.
\end{abstract}

Keywords: Caesarean myomectomy, Fibroids, Pregnancy

\section{INTRODUCTION}

Uterine leiomyomas are benign smooth muscle tumors of the uterus and found in 0.3 to $5 \%$ of pregnant women, mostly asymptomatic and may not require any surgical intervention during pregnancy and labor. ${ }^{1}$ Myomectomy during pregnancy and caesarean section is generally not advised by majority of obstetricians due to fear of intractable hemorrhage, increased incidence of miscarriage and chances of ending in hysterectomy due to severe hemorrhage and inability to obliterate tumor bed resulting in increased postoperative morbidity.

Recent studies have shown in carefully selected cases, with proper interventions to reduce the blood loss, makes myomectomy in pregnancy and caesarean section a safe and feasible procedure in experienced hands without increase in intraoperative and postoperative complications.

\section{Effects of pregnancy on fibroid}

It is a well-known clinical fact that fibroids increase in size during pregnancy which may be attributed to the hypertrophy and edema of the tumor, hyperplasia with edema and due to increased vascularity.

\section{Effects of fibroids on pregnancy}

$10-30 \%$ of women with fibroids develop complications. ${ }^{2}$ Pregnancy with a fibroid is a high-risk pregnancy which may lead to complications. Their presence has been linked to spontaneous abortion, premature labor, premature rupture of membranes, malpresentations, and malpositions, pain due to torsion, impaction or abruption or due to red degeneration, IUGR, soft tissue obstruction, increased caesarean section rates and postpartum hemorrhage. ${ }^{3}$ 
Placental abruption is increased three-fold. Submucosal fibroids, retroplacental fibroids and those large fibroids where volume is more than $200 \mathrm{~cm}^{3}$ are independent risk factors for abruption. It may be due to the diminished blood flow to the fibroid and adjacent tissues which causes partial ischemia and decidual necrosis in placenta tissue overlying leiomyoma. Large submucous fibroid can compress and distort the uterine cavity causing fetal deformities like dolicocephaly, torticollis and limb reduction defects. ${ }^{4}$

\section{Labor and delivery}

There is increased incidence of caesarean section due to malpresentation, soft tissue dystocia and also high incidence of retained placenta and postpartum hemorrhage.

\section{Diagnosis of fibroid in pregnancy}

Diagnosis in pregnancy is neither simple nor straightforward. Only $42 \%$ of large fibroids with size more than $5 \mathrm{~cm}$ and $12.5 \%$ of smaller fibroids of $3-5 \mathrm{~cm}$ can be diagnosed on physical examination. Clinically size of uterus will be more than period of gestation if fibroids are big or diagnosed before pregnancy.

The ability of ultrasound to detect fibroids in pregnancy is also limited due to difficulty in differentiating fibroids from physiological thickening of myometrium. ${ }^{5}$ Ultrasound helps in finding out size, site, number, and their relation to the placental bed.

An ultrasound study found prevalence of fibroids in pregnancy to be $18 \%$ in Africa, $8 \%$ in white women and $10 \%$ in Hispanic women. ${ }^{6}$

\section{Myomectomy in pregnancy}

It is controversial as majority of obstetricians are of the opinion to better avoid in pregnancy unless it is absolutely indicated. Recent reviews are in favor of myomectomy in pregnancy and during caesarean section in selected cases without increase in intraoperative, postoperative complications and with good maternal and perinatal outcome.

Acceptable indications for myomectomy during pregnancy. $^{7}$

1. Intractable pain during pregnancy, not relieved by conservative medical management as in cases of torsion of subserous pedunculated fibroid, anterior wall fibroids, impacted fibroids, rarely red degeneration in a large myoma.

2. To gain access to deliver the baby by lower segment caesarean section when myoma is in the lower segment.

3. Obstructed labor due to large fibroid.
4. Fibroids causing difficulty in uterine wound closure causing significant blood loss.

\section{Antepartum myomectomy}

Laparotomic myomectomy is generally avoided during ongoing pregnancy due to higher miscarriage rate and hemorrhage ending in hysterectomy. Most are performed during caesarean section. Common indications for myomectomy during pregnancy are large uterine fibroids causing renal, venous and bowel obstruction resulting in life threatening complications like renal failure, pulmonary embolism and bowel obstruction because of adhesions and bowel entrapment between the pedicle or more rarely because of pressure symptoms.

Another indication is torsion of subserous pedunculated fibroid resulting in acute abdomen.

Surgical interventions done in second trimester has better outcome. Majority of interventions are preferred laparoscopically as it is less invasive, involves minimal postoperative pain and earlier postoperative ambulation. It is suitable for small pedunculated fibroids. Large fibroids require laparotomy.

Salih et al reported a large uterine fundal fibroid with a thick pedicle measuring $30 \times 32 \mathrm{cms}$, weighing $8.9 \mathrm{~kg}$ causing intestinal obstruction was successfully managed by antepartum myomectomy at 18-20 weeks. ${ }^{8}$ At term she underwent caesarean hysterectomy due to presence of multiple myoma occupying the lower uterine segment with placenta accreta and uncontrolled blood loss from placental bed.

Domenici et al reported a case of large subserous sessile myoma of $20 \mathrm{~cm}$ who underwent myomectomy at 16 weeks of pregnancy due to intractable pelvic pain not getting relieved medically with mild bilateral dilatation of renal pelvis and extrinsic compression of ureters which was confirmed by MRI. ${ }^{9}$ Myomectomy was done with reconstruction of uterine wall. Pregnancy continued and delivered a healthy baby at 38 weeks of pregnancy weighing 3250 gms.

\section{Caesarean myomectomy}

It is usually avoided due to fear of uncontrollable hemorrhage resulting in caesarean hysterectomy. It can be safely done in selected cases with interventions to reduce blood loss.

The management of fibroids at caesarean section remains a dilemma. Recent studies have described techniques to minimize blood loss at caesarean myomectomy which include uterine tourniquet, bilateral uterine artery ligation, electrocautery and uterine artery balloon catheter. ${ }^{10}$ 
1. Application of tourniquet to encompass and compress both uterine arteries at the base of broad ligament and the vessels in infundibulopelvic ligament after lifting the fallopian tubes thus creating relatively bloodless operating field.

2. Bilateral uterine artery ligation.

3. Electrocautery.

4. Insertion of balloon catheter in uterine artery and postpartum embolisation.

5. High dose oxytocin after delivery of fetus and placenta

6. Diluted Vasopressin can be given after delivery of fetus and placenta if there is no contraindication.

What are the do's and don'ts of caesarean myomectomy. ${ }^{11}$ Points to be considered while deciding to do caesarean myomectomy or not are

1. Myomectomy during caesarean is safe, feasible and effective in carefully selected cases in tertiary care centers by experienced obstetricians. It is not always a dangerous procedure and can be performed safely without significant complications. It prevents added morbidity of a separate procedure for removal of fibroids in the future.

2. Fibroids obstructing the lower uterine segment or accessible subserosal or pedunculated fibroids in symptomatic patient can be safely removed. Removal of lower uterine segment fibroid enables lower uterine segment caesarean section, preventing classical caesarean section with its increased morbidity and high incidence of uterine rupture in future pregnancy.

3. Various hemostatic measures to decrease blood loss such as bilateral uterine artery ligation, placement of uterine artery balloon catheter, uterine tourniquets, stepwise devascularisation and post caesarean uterine artery embolisation would optimize the outcome and may reduce the choice of hysterectomy.

4. Myomectomy during caesarean section should be done in conventional way as enucleation is easier in gravid uterus owing to greater looseness of capsule. The dead spaces are obliterated with 1-0 vicryl interrupted sutures.

5. It is cost effective avoiding second surgery.

Considering these facts, the author has concluded that myomectomy during caesarean section is safe and effective.

Adesiyum et al in their study found no significant difference in hemoglobin change, incidence of postoperative complications and length of hospital stay compared to other postcaesarean patients without myomectomy. ${ }^{12}$ Fertility and obstetric outcome after caesarean myomectomy is unaffected. Many researchers have studied the feasibility of myomectomy during caesarean section either by developing new techniques for myomectomy or by devascularisation technique to reduce blood loss to prevent hysterectomy and eliminate multiple surgeries. ${ }^{13}$

Incebiyik et al in their study of 16 cases of caesarean myomectomy found it was a safe surgical procedure regardless of size and site of fibroid with no increase in intraoperative and postoperative complications. ${ }^{14}$

Apurvashri et al reported removal of twelve fibroids varying in size of 5-13 cms in six pregnant women and found no significant difference in intraoperative, postoperative complications and in length of hospital stay and with good maternal and perinatal outcome. ${ }^{15}$ Only duration of operative time was increased by 15-20 minutes. None of them required blood transfusion or hysterectomy.

Lee et al developed a new pursing suture allocated around myoma during caesarean section with the assistant maintaining strong tension on the purse-string suture around the myoma. ${ }^{16}$ They found in their study of 31 cases where caesarean myomectomy was done the purse-string suture method was effective and safe with no serious complications including late hemorrhage and uterine rupture in subsequent pregnancy.

Sapmaz et al in their study of 70 cases of caesarean myomectomy which were allocated to two groups comparing the two methods of reducing the blood loss. 52 women underwent stepwise devascularisation and 18 patients to tourniquet method. ${ }^{17}$ Intraoperative blood loss was similar in both groups, whereas postoperative blood loss was more in tourniquet method and concluded that bilateral ascending uterine artery ligation may be preferable than tourniquet.

$\mathrm{Li}$ et al in a large case control retrospective study of 1,242 pregnant women with fibroids who underwent caesarean myomectomy compared with three control groups. ${ }^{18}$ Group A with 200 pregnant women without fibroids, Group B with 145 pregnant women with fibroid who underwent only caesarean section and Group C with 51 women who underwent caesarean myomectomy and found no significant difference in mean hemoglobin change, frequency of hemorrhage, postoperative fever or length of hospital stay and concluded that caesarean myomectomy is safe, effective, feasible without any significant complications.

\section{Cervical fibroid}

Compared to uterine fibroid cervical myoma poses a challenge due to its relationship with other organs like bladder, ureter and rectum. The surgical difficulties associated with cervical myoma are poor access to myoma, increased blood loss, difficulty in suturing and repairs, distortion of the vital neighboring structures in the pelvis making it vulnerable to injuries. The pregnancy poses a unique problem because of increased vascularity making caesarean myomectomy complicated. Additional 
care has to be taken if cervical fibroid is in supravaginal portion and more than $5 \mathrm{~cm}$ size.

Chaitra et al reported a successful removal of anterior cervical fibroid of $6.5 \times 5 \mathrm{cms}$ after caesarean section after injecting diluted vasopressin. ${ }^{19}$ The interventions required to decrease the blood loss and to prevent injuries to the adjacent organs are

1. To access cervical fibroid especially anterior requires extensive bladder dissection which may be associated with massive hemorrhage in pregnancy which can be kept to minimum by staying in correct pubocervical plane and by infiltrating dilute vasopressin.

2. Intervention on uterine vessels- laparoscopic uterine artery dissection, uterine artery embolisation, paracervical mechanical tourniquet and hormonal tourniquets such as vasopressin.

3. Use of uterotonics such as ergometrine, oxytocin and misoprostol.

4. Myoma dissection techniques which include the use of laser and chemical dissectors such as sodium-2 mercaptoethane sulfonate.

5. In case of large fibroid it is better to catheterize uterine artery prophylactically and proceed with embolisation as and when necessary.

\section{Advantages of caesarean myomectomy}

1. It prevents added morbidity of an additional second procedure.

2. Prevents or decreases postpartum hemorrhage as the contraction and retraction of uterine muscle fibers which occur in postpartum occludes blood vessels and clot formation occurs in placental bed which in turn reduces bleeding.

3. Prevents classical caesarean section with its morbidity and higher incidence of uterine rupture in subsequent pregnancies by doing myomectomy of anterior cervical fibroid before lower segment caesarean section.

4. Increase chance of vaginal birth after lower segment caesarean section.

5. Cost effective.

6. Scar integrity following caesarean myomectomy has been shown to be better than interval myomectomy when assessed by serial ultrasound scans in subsequent pregnancies and subsequent caesarean section. $^{20}$

\section{Disadvantages of myomectomy in pregnancy}

1. Risk of anesthesia and surgery in antepartum myomectomy to the fetus.

2. Post-operative adhesions.

3. Increased likelihood of subsequent caesarean section due to rare risk of myomectomy scar rupture.

4. Increased cost and discomfort of two surgeries in a short period of time if done antepartum.

\section{Future obstetric performance}

It is usually good as it does not compromise uterine or ovarian blood supply. Ehigiegba et al followed up twenty-five women who underwent caesarean myomectomy and found three of them (12\%) became pregnant, two had normal vaginal delivery and one had repeat caesarean section. ${ }^{21}$

\section{CONCLUSION}

Management of fibroid uterus in pregnancy is controversial. Myomectomy in pregnancy and caesarean section is safe in carefully selected cases, by experienced obstetricians in a tertiary care center with facilities for interventions to decrease blood loss. Current evidence does not suggest routine myomectomy during pregnancy or at caesarean birth as fibroid related complications are rare and can be managed conservatively. However, in selected cases myomectomy during pregnancy and caesarean section is safe, effective and feasible option. Due to lack of multicenter randomized trials, appropriate selection criteria, surgical technique and national and gynecological society guidelines it is left to individual experience to do or not to do. What is needed is large multicenter randomized control trials to standardize the procedure with minimal complications.

Funding: No funding sources

Conflict of interest: None declared

Ethical approval: Not required

\section{REFERENCES}

1. Muram D, Gillieson MS, Walters JH. Myoma of the uterus in pregnancy. Ultrasonographic follow up. AMJ Obstet Gynecol 1980;138:16-9.

2. Katz VL, Dotters DJ, Droegemueller W. Complications of uterine leiomyomas in pregnancy. Obstet Gynecol. 1989;73:593-6.

3. Quyang DW, Economy KE, Norwitz ER. Obstetric complications of fibroids. Obstet Gynecol Clin North Am. 2006;33(1):153-69.

4. Chuang J, Tsai HW, Hwang JL. Fetal compression syndrome caused by myoma in pregnancy: a case report. Acta Obstet Gynecol Scand. 2001;80:472-3.

5. Qiidwai GI, Caughey AB, Jacoby AF. Obstetric outcomes in women with sonographically identified leiomyomata. Obstet Gynecol. 2006;107:376-82.

6. Laughlin SK, Baird DD, Savitz DA et al. Prevalence of uterine leiomyoma in first trimester of pregnancy on ultrasound screening study. Obstet Gynecol. 2009; 113:630-5.

7. Bhatla N, Dash BB, Kriplani A and Agarwal N. Myomectomy during pregnancy: a feasible option. J of Obst and Gynae Res. 2009;35(1):173-5.

8. Salih H.A.M, Sarsam RI, Abed MF, et al. Uterine fibroid causing intestinal obstruction: report of a case. J Young Pharmacists. 2015;7(4):399-402. 
9. Domenici L, Donato VDI, Gasparri ML, et al. Laparotomic myoomectomy in the 16th week of pregnancy: a case report. Case reports in Obst and Gynecology. Hindawi Publishing Corporation. 2014:154347:1-5.

10. Ortac F, Gungr M, Sommezer M. Myomectomy during caesarean section. Int $\mathbf{J}$ Gynecol Obstet 1999;67:189-90.

11. Naiknaware Sachin Vijay. Caesarean myomectomy: to do or not to do. Ecronicon open access Gynecology Editorial. EC Gynecol. 2015;2.1:114-6.

12. Adesiun AG, Ojabo A, Durosinlorun- Mohd A. Fertility and obstetric outcome after caesarean myomectomy. J Obstet Gynecol. 2008;7:710-2.

13. Holub Z, Lukac J, Kliment L, Urbnek S. Pregnancy outcome and deliveries following laparoscopic transaction of uterine vessels: a pilot study. Eur J Obstet Gynecol Reprod Biol. 2006;125(2):163-70.

14. Incebiyik A, Hilali MG, Camuzeuoglu A, Vural M, Camuzcuoglu H. Myomectomy during caesarean: a retrospective evaluation of 16 cases. Arch Gynecol Obstet. 2014;289(3):569-73.

15. Thakare A, Praneshwari Devi RK, Chowdhury PP, Deepthambiga G, Kudtarkar A. Caesarean myomectomy as safe and feasible procedure: a prospective study of 6 cases. IoSR J Dental Med Sci. 2017;16(1):17-20.
16. Lee JH, Cho DH. Myomectomy using purse-string suture during caesarean section. Arch Gynecol Obstet. 2011;283(1):35-7.

17. Sapmaz EI, Celik H, Attungul A. Bilateral ascending uterine artery ligation vs tourniquet use for hemostasis in caesarean myomectomy. J Reprod Med. 2003;48(12):950-4.

18. Li H, DUJ, Jin L, Shiz, Liu M. Myomectomy during caesarean section. Acta Obstet Gynecol Scand. 2009;88:183-6.

19. Chaitra TM, Leena GP, Urmila soman and Ratnavali Menon. Caesarean myomectomy in a cervical fibroid: A brief communication. Int J Pregn Child Birth. 2017;2(1):9.

20. Cobellis L, Messali EM, Stradella L. Myomectomy during pregnancy: different outcomes of scars. Minerva Ginecologica. 2002:54(6):483-6.

21. Ehigiegba AE, Ande AB, Ojobo SI. Myomectomy during caesarean section. Int J Gynecol Obstet. 2001;75(1):21-5.

Cite this article as: Reddi RP, Ashwini VL, John LB. Myomectomy in pregnancy: feasibility and safety. Int J Reprod Contracept Obstet Gynecol 2017;6:4204-8. 